\title{
The Role of Underlayers in EUVL
}

\author{
Pieter Vanelderen*, Danilo De Simone, Valentina Spampinato, \\ Alexis Franquet, and Geert Vandenberghe
}

\author{
IMEC, Kapeldreef 75, 3001 Leuven, Belgium \\ *Pieter.Vanelderen@imec.be
}

\begin{abstract}
There is a growing awareness that underlayers (UL) are important in EUV lithography (EUVL). In previous lithography generations ULs were required to overcome reflectivity, however that is not a concern in EUVL. Nevertheless, surface interactions are becoming prevalent because the different layers of the stack are becoming thinner. This work focuses on the influence of the spin on carbon (SOC) layer used to print line-space patterns with metal containing resists (MCR). A significant impact of underlayers on the MCR performance in EUVL is demonstrated. There are large effects on the dose to size, resist profile and stochastic nanofailures. The main contributor is the interaction between photoresist and underlayer driven by the density of the SOC UL and, to a lesser extent, other chemical interactions. The key message is that underlayer effects cannot be neglected and should be considered when evaluating the lithographic performance of photoresist materials.

Keywords: EUV, Underlayer, Metal containing resist
\end{abstract}

\section{Introduction}

Research on EUV materials is mainly focused on the development of sensitive photoresists trying to beat the RLS trade off. The role of EUV underlayers on the patterning performance of the photoresist is often neglected. UL were crucial in ArF lithography helping to overcome reflection, which does not occur in EUVL since all materials in the EUV stack have the same refractive indices (around 1) for 13.5 $\mathrm{nm}$ wavelength light. Nevertheless it is not peculiar that ULs have an effect on the EUV lithographic performance considering light-matter interactions of the UL and chemical interactions at the resistunderlayer interface [1]. Only 20 percent of the EUV photons is absorbed by a polymeric resist, meaning that a vast amount of primary and secondary electrons will be created in the underlayer. Chemical interaction and thin intermixing layers at the resist-underlayer interface will have a more pronounced effect in EUVL compared to previous lithography generations because of the increasing surface-to-volume ratios of the thin layers in the EUV stack. Typical trilayer stacks for ArF and EUV lithography are shown next to each other in Figs. 1a and $b$. It is self-evident that the thick ArF photoresist layer will relatively be less impacted by the chemistry at the resist-substrate interface compared to the thin EUVL photoresist layer. In addition to chemically amplified resists (CAR), metal containing resists (MCR) are a novel class of resist materials that have drawn a lot of attention for advanced EUV patterning for $1 \mathrm{x} \mathrm{nm}$ technology. This material has shown a good resolution and roughness performance and can compete with CAR materials [2]. MCR have a high absorption coefficient and in order to allow enough light to

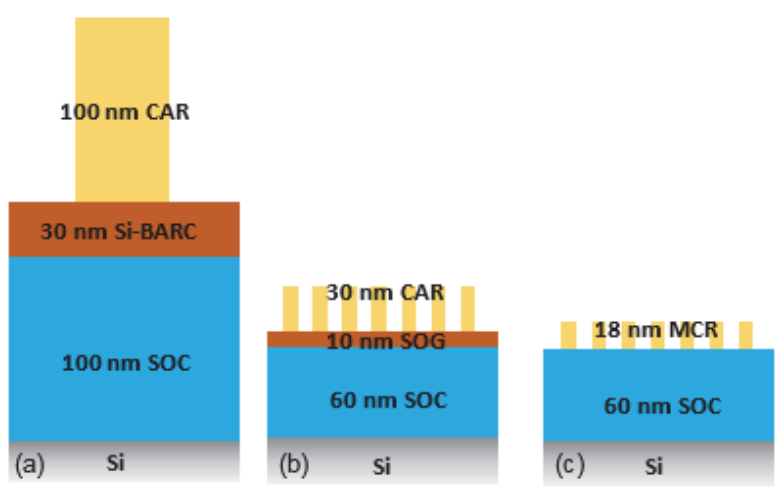

Fig. 1. Typical trilayer stack for a CAR in $\operatorname{ArF}$ (a) and EUVL (b), next to the stack for a MCR in EUVL (c). 
reach the bottom of the resist, the resist thickness is even lower than for a CAR. A typical stack is shown in Fig. 1c. Note that a MCR can be printed directly on a SOC layer, because the metal resist itself can serve as a hard mask to transfer the pattern into an organic layer. The major focus for MCR, and EUV lithography in general, is on solving the stochastic failures, mainly nano-bridges for MCR [3].

This study will be focused on the impact of the SOC layer on the lithographic performance of a MCR.

\section{Experimental}

\subsection{Patterning}

The evaluation of all underlayers is done by printing a $32 \mathrm{~nm}$ pitch 1:1 lines and space pattern using the same mask and illumination. For this patterning exercise a $300 \mathrm{~mm}$ TEL CLEAN TRACK $^{\mathrm{TM}}$ LITHIUS Pro ${ }^{\mathrm{TM}}$ interfaced with the ASML NXE3300B full field EUV scanner was used. The illumination mode was customized, similar to dipole 90X. A MCR was provided by photoresist suppliers Inpria. The thickness of the MCR is $18 \mathrm{~nm}$. Information about the underlayers and stack itself will be provided during the discussion of the results. Etch into the SOC layer was done on a Tokyo Electron (TEL) tool, the etching chemistry is not disclosed.

\subsection{Metrology}

Scanning electron microscope metrology with a Hitachi CG6300 tool was used to collect top-down images and measure line CD, $3 \sigma \mathrm{LER}$, and $3 \sigma \mathrm{LWR}$. Cross section SEM was measured with a SU-8000 microscope.

Stochastic nano-failures are quantified using IMEC in house software Stochalis [4]. This software package does an automatic assessment of top down CD-SEM images based on the brightness of the pixels. The failures are quantified with a metric pixels not $\mathrm{OK}$, which essentially represents the failure probability:

pixNOK $=\sum$ pixels in failure $/ \sum$ pixels inspected (1)

\subsection{TOF-SIMS}

Time of flight secondary ion mass spectroscopy measurements were done on a TOF-SIMS IV from ION-ToF $\mathrm{GmbH}$ that is that is equipped with a Bismuth liquid metal ion gun (LMIG) for analysis and a low energy sputter gun for sample erosion. The analysis conditions are shown in Table 1.
Table 1. Analysis conditions used for TOF-SIMS.

\begin{tabular}{ccc}
\hline & Analysis & Sputter $(<0)$ \\
\hline Beam type & Bi1 ${ }^{+}$ & $\mathrm{Cs}^{+}$ \\
\hline Beam energy & $15 \mathrm{kV}$ & $350 \mathrm{eV}$ \\
\hline Beam current & $\sim 0.5 \mathrm{pA}$ & $\sim 16 \mathrm{nA}$ \\
\hline Impact angle & $45 \mathrm{deg}$ & $45 \mathrm{deg}$ \\
\hline Raster & $100 \times 100 \mu \mathrm{m} 2$ & $400 \times 400 \mu \mathrm{m} 2$ \\
\hline
\end{tabular}

\section{Results and discussion}

3.1. Underlayer impact on the dose to size (DtS) of a metal containing photoresist (MCR)

MCRs are commonly printed directly on an SOC layer, since the resist itself serves as a hardmask [2]. In a first patterning exercise a $16 \mathrm{~nm}$ line/space pattern is printed with a MCR on a variety of SOC underlayers. Three UL suppliers provided in total 12 different SOC underlayers. Those can be divided under six different polymer types, i.e. polymer backbone. Subtle variations of side groups and crosslinking chemistry within each polymer type resulted in 12 different SOCs. A comparison of the dose sensitivity of the resist coated on the different SOC underlayers is shown in Fig. 2 with the DtS on the left y-axis. The DtS for a $16 \mathrm{~nm}$ line/space pattern of the metal containing photoresist can be modulated $40 \%$ by changing the SOC properties. It was rather unexpected that such large differences in dosing size could be obtained. The results point out the importance of carefully selecting underlayers when evaluating the photospeed of metal containing resists.

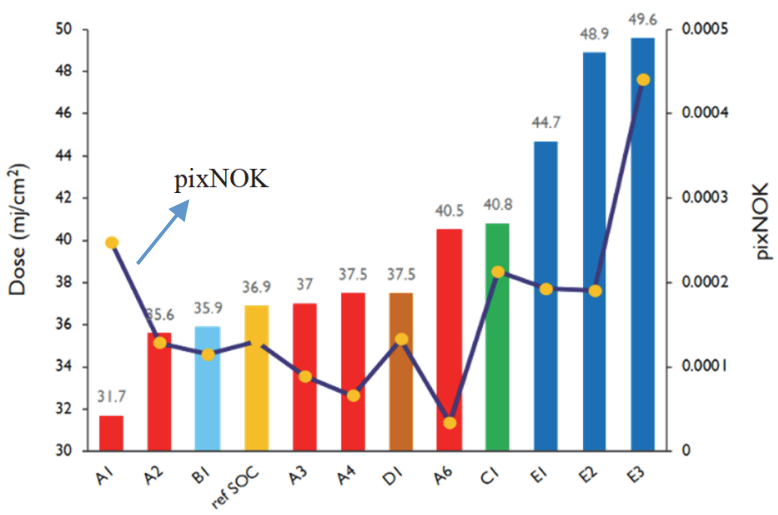

Fig. 2. DtS and pixNOK of a metal containing resist coated on a selection of different SOC underlayers, printing $16 \mathrm{~nm}$ line-space pattern. Polymers of the same type are indicated with the same color.

3.2. Underlayer impact on stochastic nanofailures of a metal containing photoresist (MCR)

Stochastic nanofailures - typically nanobridges for MCRs - are currently the main concern for single EUV exposure of $32 \mathrm{~nm}$ pitch patterns. The influence of the SOC underlayer on nanobridging defects was evaluated by using the IMEC in house 
software Stochalis. Figure 2 shows the pixNOK values on the right $y$-axis for the different underlayers. It is clear that there is modulation in amount of nanobridges when changing the SOC underlayer, however, there is no clear trend with $\mathrm{DtS}$ observed. Polymer type E clearly shows the highest number of nanobridges after litho. Some of the obtained line/space patterns were selected to be etched into the corresponding SOC layers. Figure 3 compares pixNOK before and after etch. The Stochalis analysis of the etched wafers revealed that the nanobridges, present after litho, are not transferred to the same extent into the different SOC layers. Some SOC layers even display more bridges after etch, meaning that not all failures were detected after litho. It is remarkable that the amount of nanobridges decreased drastically after etch for polymer type E. Figure 4 shows CDSEM images after etch into the polymer A1, polymer A6 and polymer E2. The difference in amount of microbridges is clearly observed in these images. In addition residues in the larger spaces can be noticed after etch into polymer A type SOCs. These residues are still present after a sufficiently long overetch and their presence depends on the nature of the substrate underneath the resist. A plausible explanation for such residues is micromasking by metal residues during etch. It is notable that the amount of such residues correlates to the amount of nanobridges that is observed after etch (Fig. 4).

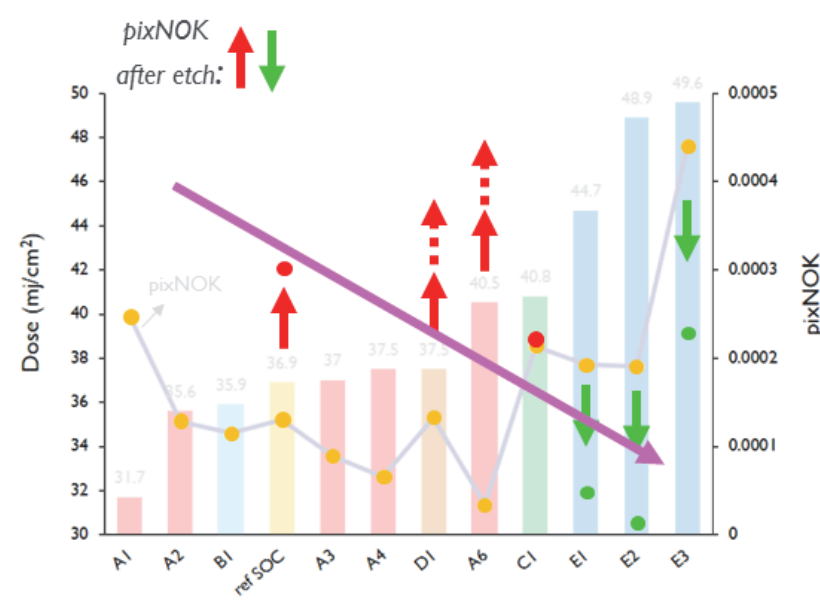

Fig. 3. Dose sensitivity and pixNOK of a metal oxide etched into a selection of different SOC underlayers, printing $16 \mathrm{~nm}$ line-space patter.
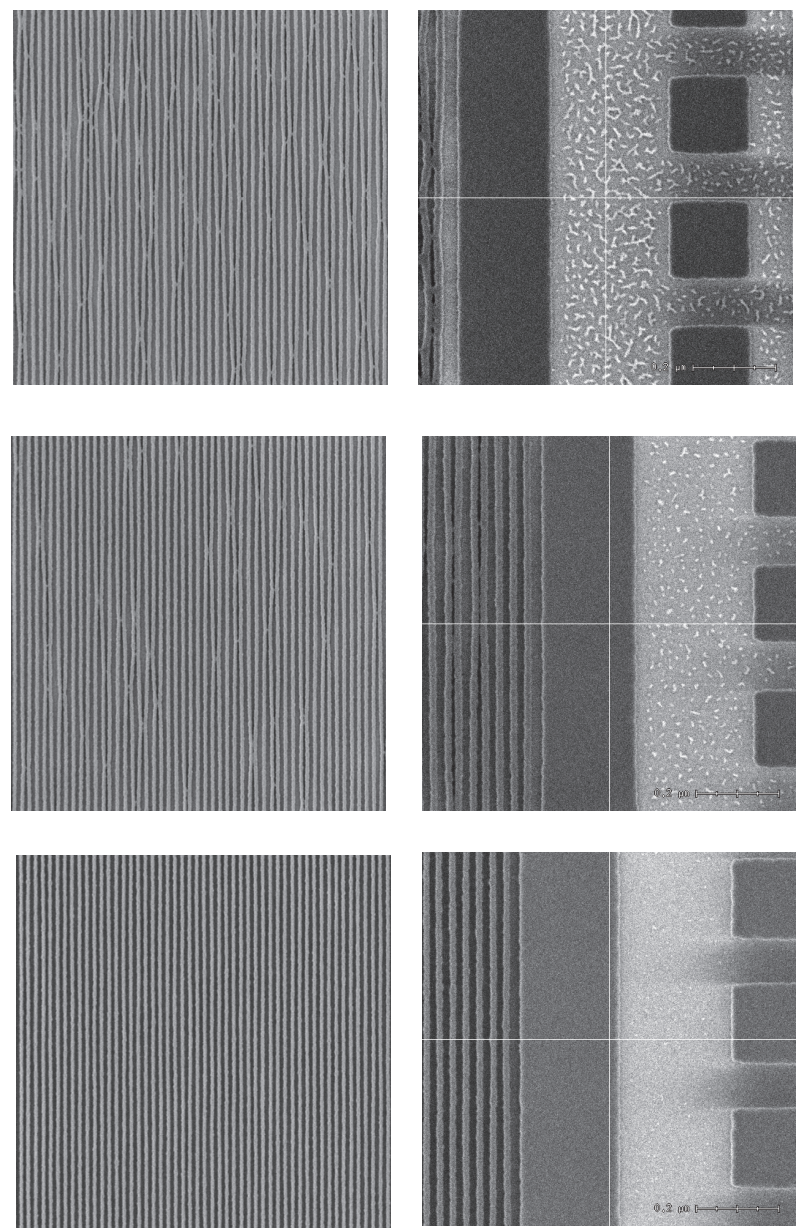

Fig. 4. $32 \mathrm{~nm}$ pitch LS - MCR resist after etch in three different underlayers, viz polymer A1 (top), polymer A6 (middle) and polymer E2 (bottom). The higher the amount of residues in the larger spaces after etch, the larger the amount of microbridges in between the lines.

Cross section SEM of the resist printed on top of polymer type A6 and polymer type E1 was measured to analyze the nanofailures into more detail. The detected failures on the cross section SEM images are shown in Fig. 5, together with top down images of similar failures. Polymer type A6 clearly causes bottom bridges, while there were no similar bottom bridges seen for polymer type E1. This indicates that the type of nanobridges is different for both underlayers.

3.3. Exploring the root causes of underlayer impact on a metal containing photoresist (MCR)

We are exploring experiments to obtain a fundamental understanding of the underlayer factors impacting resist performance. The difference in interaction between SOC and resist becomes visible when inspecting the profile of the resist lines. Figure 6 shows the profile of the resist lines printed on polymer A1, A6 and E1. The shape 

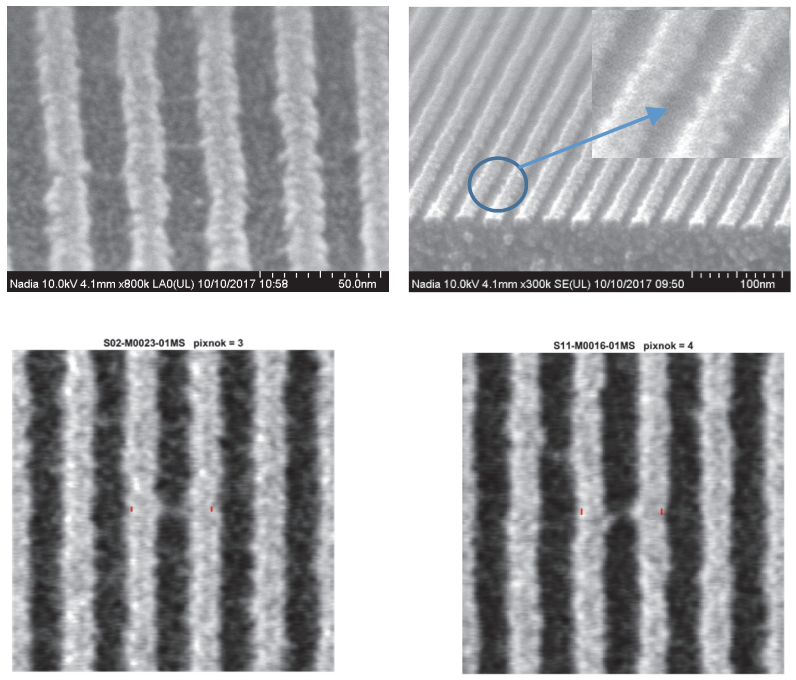

Fig. 5. Cross section SEM comparison of detected microbridges for $32 \mathrm{~nm}$ pitch LS - MCR resist on polymer A6 (left) and polymer E1 (right).
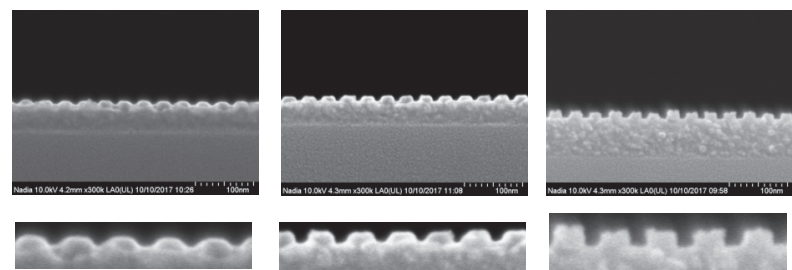

Fig. 6. Cross section SEM comparison of MCR lines profile printed on polymer A1 (left), polymer A6 (middle) and polymer E1 (right).

of the profile is entirely different for different underlayers. The lines for polymer A type SOCs have a sloped profile with a visible interacting layer between SOC and resist, while the lines on polymer E1 are of better quality and straight. A possible explanation for the sloped resist profile might be the intermixing of the resist with the UL, such an intermixing would create a small gradient of metal along the depth profile and alter the sensitivity across the resist layer. Note that the modulation of the resist profile will have a significant contribution on the DtS range (Fig. 2) that is determined form top down CD-SEM images. To a certain extent the DtS can therefore be used as probe for the resist profile and the SOC/resist interaction. The presence of such an interacting interlayer between resist and certain SOCs was experimentally tested with TOF-SIMS. SOC layers A1, A6 and E2 were coated with MCR and the resist was stripped with the developer. Subsequently the layers were analyzed with TOFSIMS to define the depth profile of the metal in the SOC layers. The Sn depth profile for the 3 SOCs is shown in Fig. 7. There is a clear modulation observed for the signal intensity of detected Sn. A large signal intensity of Sn is detected for SOC layer A1 over almost the entire depth profile, for SOC layer A6 Sn was detected in the first $10 \mathrm{~nm}$, while for SOC layer E2 almost no $\mathrm{Sn}$ was present. Although the TOF SIMS curves tend to indicate a presence of $\mathrm{Sn}$ inside the SOC, it is rather unlikely that $\mathrm{Sn}$ is still found at the bottom $(60 \mathrm{~nm})$ of the $\mathrm{SOC}$ as seen for layer A1. The Sn depth profiles should be considered with caution. The presence of Sn on top of the SOC or the different sputtering rate of the SOC and remaining Sn might cause the delayed Sn retentions seen in Fig. 7. Regardless of the mechanism causing the Sn depth profile, the TOF SIMS indicate a difference in the amount of $\mathrm{Sn}$ left on/in the SOC after coat and strip and are thus a good indication for differences in interaction between the resist and the underlayer.

The resist SOC interaction can happen during resist coating and $\mathrm{PAB}$ or during development. One can argue that during coating is more plausible giving the large effect on the DtS and the shape of the resist lines, i.e. the non developed part for this MCR negative tone resist. The TOF-SIMS results confirm the interaction of resist and SOC and, moreover, there is a correlation between the extend of interaction and the trends discussed above. A higher amount of interaction/interlayer of the resist and UL causes a lower DtS, a more sloped resist profile, more residues and a higher amount of micro bridges after SOC etch.

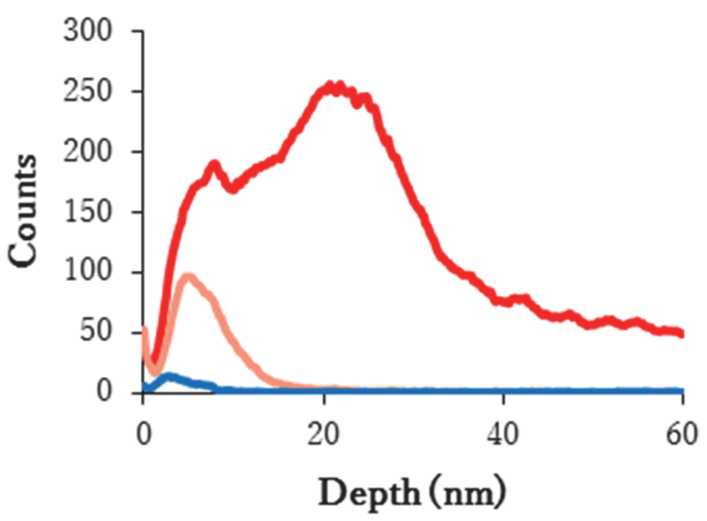

Fig. 7. Sn depth profile in SOC layer A1 (red), A6 (orange), and E2 (blue). 
3.4. SOC properties influencing resist-SOC interaction

The results discussed so far demonstrate a relation between the DtS, the resist profile, and the tendency to transfer microbridges during etch and resist-substrate interaction.

The preferred layer so far would be layer E2, since it has limited intermixing. Interestingly, Layer E2 is the most dense layer of the SOCs (see Table 2).

These results indicate that the density is a plausible main contributor in governing DtS variations and related side effects when using MCR in EUVL. This was verified by using a SOC that was designed to withstand a range of temperatures and to densify when higher bake temperatures are used. Figures $8 \mathrm{a}-\mathrm{c}$ show the thickness, mass and density of the SOC layer against the PAB temperature. MCR was coated on top of this SOC for several PAB temperatures and a $16 \mathrm{~nm}$ line-space pattern was printed. The DtS against SOC PAB temperature is shown in Fig. 8d. The DtS is increasing when the density is increased by applying a higher SOC bake temperature. High SOC density is therefore beneficial for preventing UL-resist intermixing and related side effects.

Table 2. Density of 4 selected SOC layers.

\begin{tabular}{lll}
\hline & & Denisty $(\mathrm{g} / \mathrm{cm} 3)$ \\
\hline Polymer & $\mathrm{AI}$ & $\mathrm{I} .05$ \\
\hline Polymer & $\mathrm{A} 6$ & $\mathrm{I}$ \\
\hline Polymer & $\mathrm{EI}$ & $\mathrm{I} .28$ \\
\hline Polymer & $\mathrm{E} 2$ & $\mathrm{I}$ \\
\hline
\end{tabular}

In addition, the DtS also increased when increasing the SOC PAB from $400{ }^{\circ} \mathrm{C}$ to $450{ }^{\circ} \mathrm{C}$, while the SOC density remained similar. Note that although the density didn't change the thickness and mass decreased, indicating extra chemical changes (e.g., making the SOC layer more inert) that might further impact the litho performance. Therefore, the impact of the SOC layer on the litho performance is probably driven by a combination of SOC density, being the main contributor, and other chemical interactions.
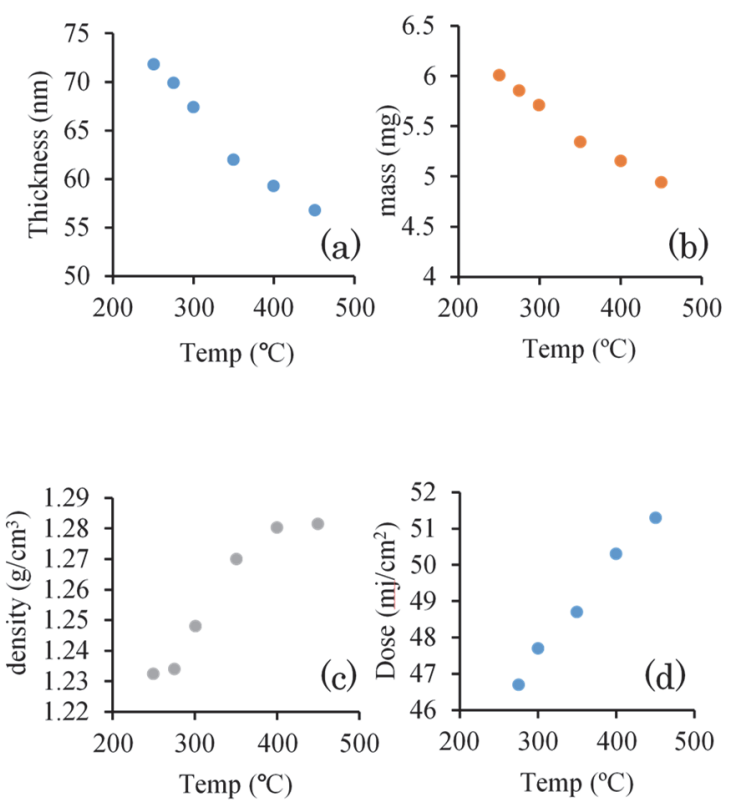

Fig. 8. Influence of the PAB temperature on the SOC layer thickness (a), mass (b) and density (c). DtS variations occur when using the SOC as UL to print 16 $\mathrm{nm} \mathrm{L} / \mathrm{S}$ patterns with MCR (d).

Cross section SEM can be used to verify if the SOC bake temperature and related density have an effect on the resist profile similar to the trend discussed in Fig. 6. Three samples with respective SOC bake temperature $275^{\circ} \mathrm{C}, 350{ }^{\circ} \mathrm{C}$ and $450{ }^{\circ} \mathrm{C}$ were selected for a cross section SEM analysis. The results are shown in Fig. 9.

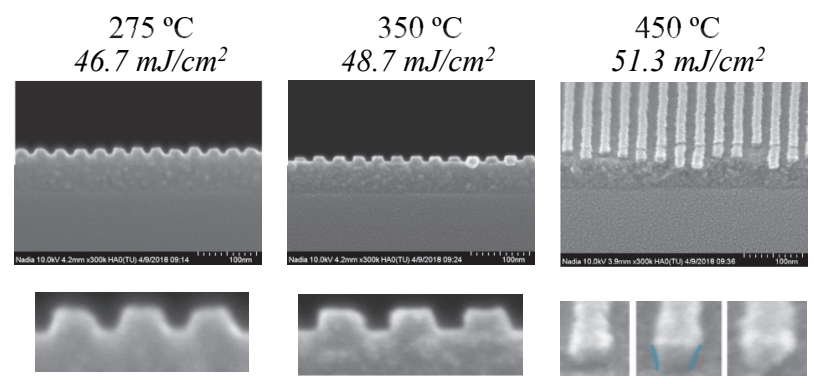

Fig. 9. Cross section SEM comparison of MCR lines profile corresponding to different SOC bake temperatures: $275^{\circ} \mathrm{C}$ (left), $350{ }^{\circ} \mathrm{C}$ (middle) and $450{ }^{\circ} \mathrm{C}$ (right).

The resist profiles follow the expected trend, i.e. a lower DtS corresponds to a sloped resist profile while a higher DtS to a straight resist profile. Remarkably the highest SOC bake temperature, with a DtS of $51.3 \mathrm{~mJ} / \mathrm{cm}^{2}$, has an inversed resist profile. Such a profile is in fact what one would expect for a highly absorbing NTD resist, 
nevertheless, it was only observed for this sample.

Finally, the observed density related trend is confirmed considering a plot (Fig. 10) of the DtS vs. SOC density of several different SOCs used in this study. A clear impact of the UL density on the DtS becomes visibly.

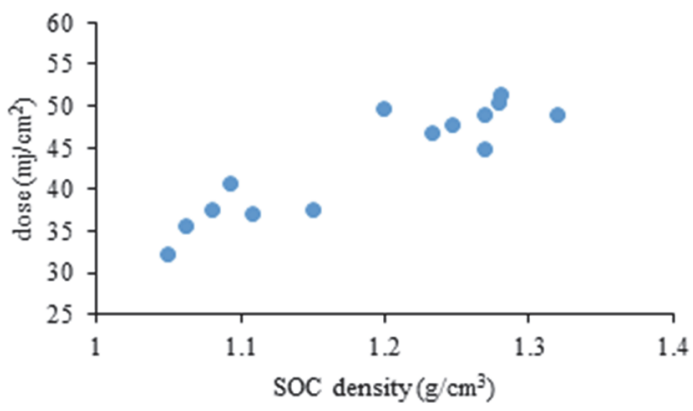

Fig.10. DtS for printing $16 \mathrm{~nm}$ lines and space with a MCR plotted against the SOC density underneath the resist.

\section{Conclusion}

EUVL is making use of very thin photoresist layers, drastically decreasing the bulk to surface ratio of a photoresist layer. Therefore interactions at interfaces will have a relatively larger impact on the photoresist compared to previous lithography generations. In this work clear evidence of UL impact on MCR performance is reported.

There is a large influence of the SOC on the DtS, resist profile, amount of nanofailures and the extend at which those transfer during SOC etch. It became clear that the density and to a lesser extend the chemical properties of the SOC play an important role when using MCR in EUVL. Therefore the underlayer should always be compatible with the photoresist.

It will become important to consider the influence of the UL when comparing and evaluating photoresists or building photoresist models. All together these findings will create many new opportunities for material suppliers to develop underlayers that enhance the lithographic performance, enabling future node processing.

\section{Acknowledgement}

The authors would like to thank Brewer Science and Nissan Chemical Industries, for their participation this EUV underlayer exploration activity and Inpria for providing the MCR.

\section{References}

1. D. L. Goldfarb, M. Glodde, A. De Silva, I. Seshadri, N. M. Felix, K. Lionti, and T. Magbitang, Proc. SPIE, 10146 (2017) 1014607.

2. D. De Simone, I. Pollentier, and G. Vandenberghe, J. Photopolym. Sci. Technol., 28 (2015) 507.

3. D. De Simone, P. Vanelderen, and G. Vandenberghe, J. Photopolym. Sci. Technol., 30 (2017) 613.

4. P. De Bisschop and E. Hendrickx, Proc. SPIE, $10583 \quad$ (2018) 105831K, doi: $10.1117 / 12.2300541$ 